\title{
SUR UNE CLASSE D'INTÉGRALES DOUBLES
}

PAR

\author{
E. GOURSAT \\ a TOULOUSE.
}

Le présent mémoire n'est qu'une suite d'un travail publié antérieurement dans le même recueil, $\left({ }^{1}\right)$ où j'indiquais une méthode générale pour l'étude des coupures des intégrales définies. Je m'occupe plus spécialement des intégrales doubles qui ont été signalées par M. Hermite à la fin de sa lettre à M. Mittag-Leffler (Journal de Borchard, tome 91).

I. L'artifice que j'emploie étant tout-à-fait général, je commencerai par le rappeler en quelques mots. Soit en premier lieu $f(x, u)$ une fonction uniforme ou multiforme des deux variables indépendantes $x$ et $u$, telle que lorsqu'on attribue à $x$ une valeur particulière $x_{1}$, les valeurs de $u$ qui sont des valeurs singulières pour $f\left(x_{1}, u\right)$ ne forment ni des lignes ni des surfaces, ces valeurs pouvant d'ailleurs être en nombre fini ou infini. Appelons $\Phi(x)$ l'intégrale

$$
\int_{u_{0}}^{u_{1}} f(x, u) d u,
$$

prise suivant la ligne droite qui joint les deux points $u_{0}, u_{1}$. Ayant adopté pour $f\left(x, u_{0}\right)$ une quelconque de ses déterminations, l'intégrale précédente représente une fonction qui peut être uniforme ou multiforme, mais qui conserve un sens bien déterminé tant que le chemin décrit par

( ) Voir Acta Mathematica, T. 2, p. I-70. 
E. Goursat.

la variable $x$ ne rencontre pas certaines lignes qui sont des coupures pour cette intégrale. Pour savoir ce que devient cette fonction lorsque la variable vient à rencontrer une de ces lignes, je procède de la manière suivante. Soit $C$ une coupure et admettons que lorsque $x$ vient coïncider avec un point $\xi$ situć sur cette ligne, la fonction $f(\xi, u)$ est discontinue pour un point $\theta$ situé sur la ligne $u_{0} u_{1}$ et reste finie pour toute autre

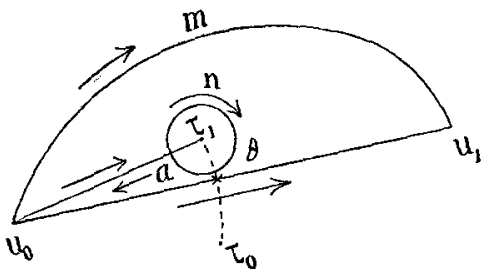

Fig. 1

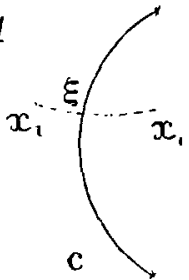
valeur de $u$ sur ce chemin (fig. I); de sorte que $x$ allant de $x_{0}$ en $x_{1}$, le point qui coïncide avec $\theta$ pour $x=\xi$ va de $\tau_{0}$ en $\tau_{1}$ en traversant la ligne $u_{0} u_{1}$. La méthode consiste uniquement à remplacer l'inté-

grale $\int_{u_{0}}^{u_{1}} f(x, u) d u$ qui cesse d'avoir un sens par la même intégrale prise sur le chemin infiniment voisin $u_{0} m u_{1}$ qui est égale à la première et qui continue à avoir un sens. De sorte que lorsque $x$ est venue en $x_{1}$, la continuation analytique de $\Phi(x)$ se trouve représentée par le méme symbole ou par la somme des deux intégrales

$$
\int_{u_{0} \times r u u_{0}} f(x, u) d u+\int_{u_{0}}^{u_{1}} f(x, u) d u \text {. }
$$

$\mathrm{Au}$ lieu de supposer que la fonction $f(x, u)$ n'admet que des points singuliers isolés, on peut se proposer de considérer le cas où cette fonction admet elle-mème des coupures ou mème des espaces lacunaires. Supposons, par exemple, que lorsqu'on attribue à $x$ une valeur particulière
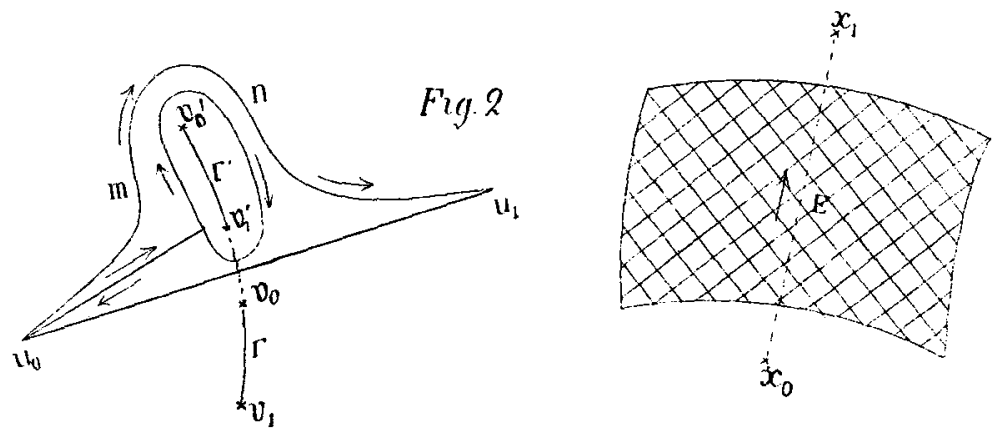
$x_{1}, f\left(x_{1}, u\right)$ admette une ligne de discontinuité, ligne qui est elle-mème variable avec $x$, de façon que lorsque $x$ est compris dans une certaine portion du plan $E$, cette coupure a un ou plusieurs points communs avec la ligne droite $u_{0} u_{1}$.

L'intégrale $\Phi(x)=\int_{u_{0}}^{u_{1}} f(x, u) d u$ n'aura aucun sens pour toute valeur de $x$ comprise dans cette portion du plan (fig. 2).

Admettons, pour fixer les idées, que lorsque $x$ traverse l'espace $E$ suivant la ligne $x_{0} x_{1}$, la coupure variable $\Gamma$ traverse la ligne $u_{0} u_{1}$ sans qu'aucun de ses points vienne coïncider avec une des extrémités $u_{0}, u_{1}$, ct passe de la position primitive $v_{0} v_{1}$ à la nouvelle position $v_{0}^{\prime} v_{1}^{\prime}$. On peut, par l'application répétée du théorème de $\mathrm{C}_{\Lambda \mathrm{UCHY}}$, supposer que le chemin d'intégration se déforme d'une manière continue, de façon que la coupure ne l'atteigne jamais; et, lorsque $x$ sera venue en $x_{1}$ la continuation analytique de $\Phi(x)$ sera représentée par la mème intégrale prise suivant le chemin $u_{0} m n u_{1}$. Si la fonction $f(x, u)$ est uniforme à l'intérieur du contour formé par ce chemin et la ligne droite $u_{0} u_{1}$, on pourra remplacer cette intégrale par l'intégrale primitive $\Phi(x)$, augmentéc de l'intégrale

$$
\int f(x, u) d u
$$

prise dans le sens inverse le long d'un contour fermé environnant complètement la ligne $v_{0}^{\prime} v_{1}^{\prime}$.

2. Cette généralisation de la méthode conduit bien aisément, comme on le verra plus loin, à des conséquences importantes relatives aux intégrales doubles; mais avant d'aborder ce point, il est nécessaire d'étudier de plus près les propriétés des intégrales simples analogues à celles de M. Henmite. Soit $f(x, u)$ une fonction jouissant des propriétés qui viennent d'ètre rappelées et $\alpha_{0} \alpha_{1}$ une coupure de l'intégrale

$$
\int f(x, u) d u
$$

prise suivant une ligne quelconque telle que $u_{0} u_{1}$ (fig. 3 ).

Je suppose que la coupure $\alpha_{0} \alpha_{1}$ ne se coupe pas elle-mème, et ne rencontre aucune autre coupure relative à la même intégrale. Lorsque $x$ vient coïncider avec un point de cette ligne, $f(x, u)$ est discontinue 
pour un seul point de la ligne $u_{0} u_{2}$. Soit $v=\phi(x)$ la valeur de $u$ qui est pour $f(x, u)$ une valeur singulière; nous pourrons entourer la ligne $\alpha_{0} \alpha_{1}$ d'un contour suffisamment voisin $C$ pour que la fonction $\psi(x)$ soit
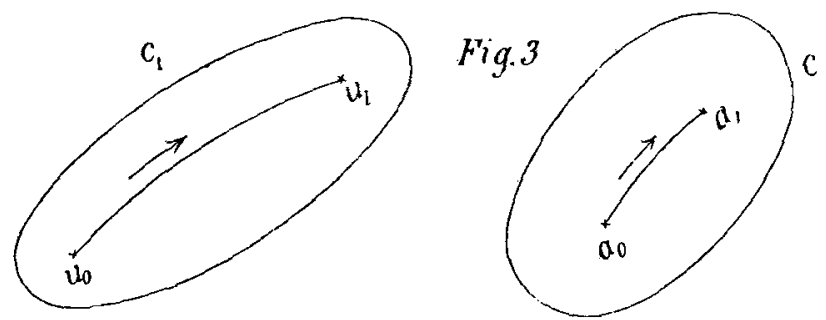

uniforme à l'intérieur de ce contour. Le point $x$ décrivant la ligne $\alpha_{0} \alpha_{1}, v$ décrit la ligne $u_{0} u_{1}$. Inversement lorsque $v$ décrit la ligne $u_{0} u_{1}$, il $\mathrm{y}$ a une seule valeur de $x$ déduite de la relation

$$
v=\psi(x)
$$

qui soit située sur la ligne $\alpha_{0} \alpha_{1}$, puisque cette ligne ne rencontre aucune autre des coupures. Cela revient à supposer que la fonction $x$ de $v$ définie par l'équation $v=\phi(x)$ est uniforme à l'intérieur d'un certain contour $C_{1}$ entourant $u_{0} u_{1}$. Les points des deux lignes $u_{0} u_{1}, \alpha_{0} \alpha_{1}$ se correspondent donc un $\dot{a}$ un, et sont décrits simultanément dans le sens indiqué par les flèches. On comprendra suffisamment, sans qu'il soit besoin de le définir, ce que j'entendrai désormais par bord droit et bord gauche de la coupure $\alpha_{0} \alpha_{1}$. Je suppose encore que $f(x, u)$ est uniforme pour les valeurs de $x$ et de $u$ comprises à l'intérieur des contours $C$ et $C_{1}$; de sorte que le point $v=\psi(x)$ est pour cette fonction un pôle ou un point singulier essentiel. J'appelle $R(x)$ le résidu relatif à ce point singulier; $R(x)$ est comme $\phi(x)$ une fonction uniforme à l'intérieur de $C$. Ces hypothèses étant adoptées, l'intégrale

$$
\Phi(x)=\int_{u_{0}}^{u_{1}} f(x, u) d u
$$

représente une fonction uniforme de $x$ à l'intérieur de $C$ qui admet la ligne $\alpha_{0} \alpha_{1}$ comine coupure. On reconnait, d'après la méthode générale rappelée au début, que lorsque $x$ traverse la coupure $\alpha_{0} \alpha_{1}$, la fonction 
reste continue, et se trouve représentée après le passage par la même intégrale définie augmentée de la quantité $\pm 2 i \pi R(x)$, le signe + ou devant être pris suivant qu'on passe $d u$ bord gauche au bord droit ou inversement. De sorte qu'en deux points infiniment voisins d'un point $\xi$ de la ligne $a_{0} \alpha_{1}$, on a

$$
\phi(N)-\phi\left(N^{\prime}\right)=2 i \pi R(\xi)
$$

le point $N$ étant pris sur le bord gauche. On voit en outre que la fonction $\Phi(x)$ ne présente à l'intérieur du contour $C$ que deux points véritablement singuliers $\alpha_{0}, \alpha_{1}$, et qu'elle peut ètre mise sous la forme

$$
R(x) L\left(\frac{x-\alpha_{1}}{x-u_{0}}\right)+P(x)
$$

$P(x)$ étant une fonction uniforme dans la mème région du plan qui ne peut présenter de discontinuité qu'aux points $\alpha_{0}$ et $\alpha_{1}$.

3. Pour reconnaitre quelle est la forme de cette fonction $P(x)$ dans le voisinage de ces points singuliers, je prends d'abord le cas où le point $v=\psi(x)$ est un pôle simple pour la fonction $f(x, u)$. La différence

$$
f(x, u)-\frac{R(x)}{u-\psi(x)}
$$

sera une fonction holomorphe à l'intérieur des contours $C$ et $C_{1}, Q(x, u)$; et on aura

$$
\begin{aligned}
\int_{u_{0}}^{u_{1}} f(x, u) d u & =\int_{u_{0}}^{u_{1}} \frac{R(x) d u}{u-\psi(x)}+\int_{u_{0}}^{u_{1}} Q(x, u) d u \\
& =R(x) L\left\{u_{1}-\psi(x)\right\}+\left[\int_{u_{0}}^{u_{1}} Q(x, u) d u-R(x) L\left\{u_{0}-\psi(x)\right\}\right]
\end{aligned}
$$

La seconde partie est continue dans le voisinage du point $x=\alpha_{1}$; de plus, comme $\psi(x)$ devient égal ’̀ $u_{1}$ pour $x=\alpha_{1}$, on a dans le voisinage de ce point

$$
\phi(x)=u_{1}+A_{1}\left(x-\alpha_{1}\right)+A_{2}\left(x-\alpha_{1}\right)^{2}+\ldots
$$


$A_{1}$ étant différent de zéro puisqu'on suppose que la coupure $\alpha_{0} \alpha_{1}$ est isolée ou qu'une seule valeur de $x$ devient égale à $\alpha_{1}$ pour $u=u_{1}$. Ceci peut s'écrire

$$
u_{1}-\phi(x)=\left(x-\alpha_{1}\right) \psi_{1}(x),
$$

$\psi_{1}(x)$ désignant une fonction holomorphe pour $x=\alpha_{1}$, et différente de zéro pour cette valeur. La première partie est donc égale à

$$
R(x) L\left(x-a_{1}\right)+R(x) L\left\{\phi_{1}(x)\right\}
$$

ce qui montre que la fonction $P(x)$ est uniforme et continue dans le domaine du point $\alpha_{1}$, et il en est évidemment de mème dans le domaine da point $\alpha_{0}$.

Plus généralement supposons que le point $v=\psi(x)$ soit pour $f(x, u)$ un póle d'ordre $n$, de telle sorte qu'en retranchant de cette fonction une expression de la forme

$$
\frac{B_{1}}{[u-\psi(x)]^{n}}+\frac{B_{9}}{[u-\psi(x)]^{n-1}}+\ldots+\frac{B_{n-1}}{[u-\psi(x)]^{2}}+\frac{R(x)}{u-\psi(x)},
$$

où les $B$ sont des fonctions uniformes de $x$, le résultat soit une fonction uniforme de $x$ et de $u$. On a pour l'intégrale considérée la mème expression que tout-à-l'heure sauf les termes

$$
-\frac{\mathrm{I}}{n-\mathrm{I}} \frac{B_{1}}{\left[u_{1}-\psi(x)\right]^{n-1}}-\ldots \cdot-\frac{B_{n-1}}{u_{1}-\psi(x)}
$$

or dans le voisinage du point $x=\alpha_{1}$, on a en général

$$
\frac{\mathbf{I}}{\left[u_{1}-\psi(x)\right]^{m}}=\frac{\mathbf{I}}{\left(x-u_{1}\right)^{m}} \phi_{2}(x),
$$

$\psi_{2}(x)$ étant une fonction holomorphe de $x$ pour $x=\alpha_{1}$; de sorte que ce point $\alpha_{1}$ sera en général pour l'intégrale un pôle d'ordre $n-\mathrm{I}$. On verrait de mème que si le point $v=\psi(x)$ est pour $f(x, u)$ un point singulier essentiel, il en est de même des points $\alpha_{0}$ et $\alpha_{1}$ pour $P(x)$.

Ainsi, dans les intégrales de M. Hermite

$$
\Phi(x)=\int_{u_{0}}^{u_{1}} \frac{F(x, u)}{G(x, u)} d u,
$$


où $F(x, u), G(x, u)$ sont des fonctions entières de $x$ et de $u$, les points $\alpha_{0}$ et $\alpha_{1}$ seront des points ordinaires pour $P(x)$, tandis que dans les intégrales

$$
\Phi_{1}(x)=\int_{u_{0}}^{u_{1}} \frac{F(x, u)}{G^{2}(x, u)} d u
$$

ces mêmes points seront des pôles simples pour $P(x)$. Cherchons le résidu relatif à ces pôles. On a

$$
\frac{\mathbf{I}}{G(x, u)}=\frac{\mathbf{I}}{[u-\varphi(x)] \frac{\partial G}{\partial u}}+Q(x, u)
$$

$F(x, u)=F(x, \phi(x))+F(x, u)-F(x, \phi(x))=F(x, \phi(x))+[u-\psi(x)] Q_{1}(x, u)$,

$Q$ et $Q_{1}$ étant des fonctions holomorphes de $x$ et de $u$ et en supposant que dans $\frac{\partial G}{\partial u}$ on ait remplacé $u$ par $\phi(x)$. On aura alors

$$
\frac{F(x, u)}{G^{2}(x, u)}=\frac{F(x, \phi(x))}{[u-\psi(x)]^{2}\left(\frac{\partial G}{\partial u}\right)^{2}}+\frac{R(x)}{u-\psi(x)}+Q_{2}(x, u)
$$

$Q_{2}(x, u)$ étant également une fonction holomorphe de $x$ et de $u$; par suite, dans le voisinage de $x=x_{1}$, on aura

$$
\Phi_{1}(x)=-\frac{F(x, \psi(x))}{\left[u_{1}-\psi(x)\right]\left(\frac{\partial G}{\partial u}\right)^{2}}+R(x) L\left(x-\alpha_{1}\right)+P_{1}\left(x-\alpha_{1}\right)
$$

$P_{1}\left(x-a_{1}\right)$ étant continue dans le domaine du point $a_{1}$. Mais dans ce même domaine on a

$$
\begin{gathered}
\psi(x)=u_{1}+\frac{d \psi}{d x}\left(x-\alpha_{1}\right)+\ldots \\
\frac{\partial G}{\partial u} \frac{d \psi}{d x}+\frac{\partial G}{\partial x}=0
\end{gathered}
$$


par suite

$$
\frac{\mathrm{I}}{\phi(x)-u_{1}}=\frac{\mathrm{I}}{\left(x-a_{1}\right) \frac{d \phi}{d x}+\cdots}=\frac{-\frac{\partial G}{\partial u}}{\cdot\left(x-a_{1}\right) \frac{\partial G}{\partial x}+\cdots}
$$

et le résidu relatif au pôle $\alpha_{1}$ sera évidemment

$$
\frac{-F\left(\alpha_{1}, u_{1}\right)}{\frac{\partial G}{\partial u}} \frac{\partial G}{\partial x}
$$

en supposant que dans $\frac{\partial G}{\partial u}$ et $\frac{\partial G}{\partial x}$ on fasse $x=\alpha_{1}, u=u_{1}$. Le résidu relatif au pôle $\alpha_{0}$ aurait pour expression

$$
\frac{F\left(a_{0}, u_{0}\right)}{\frac{\partial G}{\partial u} \frac{\partial G}{\partial x}} .
$$

4. Comme application, soit $f(x)$ une fonction uniforme et continue le long d'un arc de courbe $C$ : l'intégrale

$$
\Phi(x)=\int_{C} \frac{f(u) d u}{u-x}
$$

prise le long de $C$, définit une fonction de $x$ holomorphe dans toute l'étendue du plan, sauf sur cette courbe qu'elle admet comme coupure, la différence des valeurs de $\Phi(x)$ en deux points infiniment voisins d'un point $\xi$ de part et d'autre de cette ligne étant précisément égale à $2 i \pi . f(\xi)$. En ajoutant plusieurs intégrales de cette nature, on parvient à l'expression, sous forme d'intégrale définie, d'une fonction holomorphe dans toute l'étendue du plan, snuf le long de certains arcs de courbe $C_{1}, C_{2}, \ldots, C_{n}$, la différence des valeurs en deux points infiniment voisins d'une coupure étant égale à $f_{1}(x)$ pour $C_{1}$, ̀̀ $f_{2}(x)$ pour $C_{2}, \ldots, f_{n}(x)$ pour $C_{n}, f_{1}, f_{2}, \ldots, f_{n}$ désignant des fonctions tout-à-fait arbitraires. Dans le cas on la ligne d'intégration se compose d'un arc de cercle, on retombe sur des intégrales qui se sont présentées à un autre point de vue dans le beau mémoire de M. Appexx Sur le développement d'une fonction holomorphe dans une aire limitée par des arcs de cercle (Acta Mathematica, T. I, p. I 47). 
5. Je considère encore l'intégrale double suivante sur laquelle M. Hermite a appelé mon attention:

$$
\Phi(x)=\int_{u_{0}}^{u_{1}} \int_{i_{0}}^{t_{1}} \frac{F(x, u, t) d u d t}{G(x, u) G_{1}(x, t)}
$$

où $F(x, u, t), G(x, u), G_{1}(x, t)$ désignent, pour fixer les idées, des fonctions entières des variables qui y figurent et où les intégrations sont effectuées suivant des lignes droites. Cette intégrale admet deux sortes de coupures; elles s'obtiennent en prenant, dune part, la série des valeurs de $x$ que l'équation $G(x, u)=0$ fait correspondre à la série des valeurs de $u$ croissant de $u_{0}$ à $u_{1}$ et, d'autre part, la série des valeurs de $x$ qui correspondent à la série des valeurs de $t$ croissant de $t_{0}$ à $t_{1}$ d'après l'équation $G_{1}(x, t)=0$. Soient $C_{u}$ les premières et $C_{t}$ les secondes, que nous supposons distinctes les unes des autres. Je considere par exemple une coupure $C_{u}$ et je prends sur cette coupure un point $\xi$, n'appartenant pas à une coupure $C_{t}$ et n'étant pas un point double pour la coupure $C_{u}$; de telle sorte qu'une seule des valeurs de $u$ déduite de l'équation

$$
G(\xi, u)=\mathrm{o}
$$

soit située sur le chemin $u_{0} u_{1}$; soit $\theta$ le point qui figure cette valeur de $u$. Posons

$$
I(x, u)=\int_{i_{0}}^{t_{1}} \frac{F^{\top}(x, u, t) d t}{G_{1}(x, t)}
$$

$\eta(x, u)$ est évidemment une fonction holomorphe de $x$ et de $u$, tani que $x$ reste compris dans les environs du point $\xi$. On peut alors répéter mot pour mot pour l'intégrale

$$
\phi(x)=\int_{u_{0}}^{u_{1}} \frac{\Pi(x, u) d u}{G(x, u)}
$$

le raisonnement qui a été fait pour les intégrales de M. Hermite, et on 
en déduit que la différence $\Phi(N)-\Phi\left(N^{\prime}\right)$ est égale à $2 i \pi$ multiplié par le résidu de la fonction

$$
\frac{\Pi(\xi, u)}{\bar{G}(\xi, u)}
$$

relatif au pôle $u=\theta, N$ et $N^{\prime}$ étant deux points infiniment voisins de $\xi$, $N$ sur le bord gauche et $N^{\prime}$ sur le bord droit de la coupure considérée. Ce résidu a pour expression

$$
\frac{\Pi(\xi, \theta)}{\frac{\partial}{\partial \theta} G(\xi, \theta)}
$$

on aura donc, en remplaçant $I$ par sa valeur,

$$
\Phi(N)-\Phi\left(N^{\prime}\right)=2 i \pi \int_{i_{0}}^{t_{1}} \frac{F(\xi, \theta, t) d t}{\frac{\partial}{\partial \theta}\{G(\xi, \theta)\} \cdot G_{1}(\xi, t)},
$$

et on trouverait pour la différence des valeurs de $\Phi(x)$ en deux points infiniment voisins d'une coupure $C_{t}$ une expression analogue.

6. Les intégrales doubles signalées par M. Hermite sont de la forme

$$
\int_{u_{0}}^{u_{1}} d u \int_{t_{0}}^{t_{1}} \frac{F(x, u, t) d t}{G^{2}(x, u, t)} ;
$$

je considère, pour plus de généralité, les intégrales de la forme

$$
\Phi(x)=\int_{u_{0}}^{u_{1}} d u \int_{t_{0}}^{t_{1}} \frac{F(x, u, t) d t}{G^{n}(x, u, t)},
$$

où $F(x, u, t), G(x, u, t)$ désignent des fonctions entières de $x, u, t$, où $n$ est un nombre entier positif et où les intégrations sont effectuées suivant des lignes droites. Le symbole $\Phi(x)$ offre un sens bien déterminé, sauf pour les valeurs de $x$ que l'on obtient en faisant varier $u$ de $u_{0}$ à $u_{1}$ et $t$ de $t_{0}$ à $t_{1}$ suivant des lignes droites dans l'équation

$$
G(x, u, t)=\mathrm{o} .
$$


L'ensemble de ces valeurs de $x$ recouvre en général une certaine portion du plan que j'appelle $E$; de sorte que la définition de la fonction $\phi(x)$ par une intégrale double ne nous apprend rien sur le mode d'existence de cette fonction à l'intérieur de l'espace $E$. Il n'en résulte pas que cette portion du plan soit pour la fonction $\phi(x)$ un espace lacunaire, au sens attribué à ce mot depuis les travaux de M. Weierstrass et de ses disciples. Soit $x_{0}$ un point extérieur à $E$, mais très-voisin de la limite; la fonction considérée étant holomorphe pour $x=x_{0}$, il existe comme on sait une série procédant suivant les puissances croissantes et positives de $x-x_{0}$, convergente à l'intérieur d'un cercle ayant pour centre le point $x_{0}$, et dont la somme est égale à $\Phi(x)$ pour tous les points du cercle extérieurs à l'espace $E$. Deux cas peuvent se présenter: ou bien ce cercle ne pénètre pas à l'intérieur de $E$, quel que soit le point $x_{0}$, et alors $E$ est bien véritablement un espace lacunaire; ou bien ce cercle pénètre à l'intérieur de $E$. Nous allons reconnaitre que c'est ce qui a lieu dans l'exemple considéré; de telle manière que la fonction qui coïncide avec $\Phi(x)$ à l'extérieur de $E$ peut être continuée analytiquement dans cette région. Nous verrons de plus qu'on peut atteindre ainsi tous les points de cette région, sauf ún certain nombre de points isolés.

Pour plus de clarté, je me bornerai à l'étude d'un cas simple. Je suppose que, $u$ et $t$ variant dans les limites indiquées, une des valeurs de $x$ déduite de l'équation

$$
G(x, u, t)=\mathrm{o}
$$

vienne coïncider une fois et une seule fois avec chacun des points ̀̀ l'intérieur d'une portion du plan simplement connexe, que je désignerai dorénavant par $E$; de telle sorte qu'en prenant un contour $C$, suffisamment rapproché de la limite de $E$, toutes les autres valeurs de $x$, déduites de l'équation (3), soient figurées par des points à l'extérieur de $C$. Le symbole $\Phi(x)$ définit alors une fonction holomorphe de $x$ entre la limite de $E$ et le contour $C$.

Cet espace $E$ peut être envisagé comme une espèce de quadrilatère curviligne. Si, dans l'équation (3), on attribue à $t$ la valeur $t_{0}$ et qu'on fasse varier $u$ de $u_{0}$ à $u_{1}$, la valeur correspondante de $x$, comprise à l'intérieur de $C$, décrit un certain arc de courbe que je désignerai par $T_{0}$, 
et j'appellerai de même $T_{1}, U_{0}, U_{1}$ les trois autres arcs de courbe engendrés d'une façon analogue, en remarquant que deux arcs désignés par deux lettres différentes ont toujours une extrémité commune et que deux arcs désignés par la même lettre n'ont aucun point commun. Ces arcs forment par conséquent les côtés d'un quadrilatère curviligne convexe. Plus généralement, soit $\theta$ un point du segment de droite $t_{0} t_{1}$, et appelons $T_{\theta}$ l'arc de courbe décrit par la variable $x$ lorsque dans l'équation $G(x, u, \theta)=0$ on fait varier $u$ de $u_{0} \grave{a} u_{1}$. Lorsque le point $\theta$ passe de la position $t_{0}$ à la position $t_{1}$, cet arc de courbe $T_{\theta}$ se déplace en se déformant d'une manière continue de façon que deux arcs différents ne se coupent jamais et que ses extrémités décrivent les côtés $U_{0}, U_{1}$. Il passe ainsi de la position primitive $T_{0}$ à la position $T_{1}$, après avoir décrit une fois et une seule fois tout l'espace $E$. Tout pareillement on peut concevoir que l'arc de courbe $U_{0}$ passe d'une manière continue de la position primitive à la position $U_{1}$. Ce double mode de génération se trouve mis en évidence par la figure suivante (fig. 4).

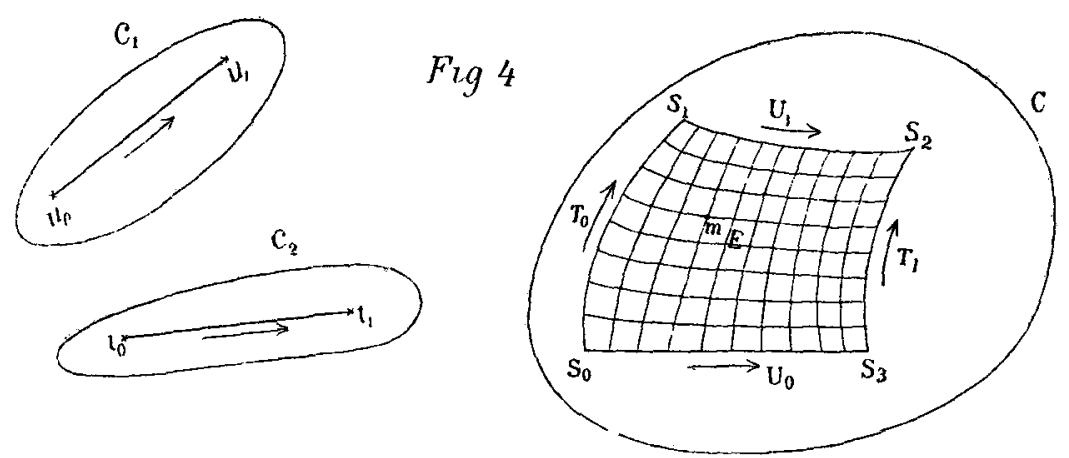

Par chaque point $m$ de $E$ passe une seule courbe $T_{\theta}$ et une seule courbe $U_{\lambda}$; ̀̀ chaque point tel que $m$ correspond ainsi un seul couple de points dont l'un est sur $t_{0} t_{1}$, l'autre sur $u_{0} u_{1}$. Par exemple les sommets $S_{0}, S_{1}, S_{2}, S_{3}$ du quadrilatère correspondent respectivement aux couples $\left(u_{0}, t_{0}\right),\left(u_{1}, t_{0}\right),\left(u_{1}, t_{1}\right),\left(u_{0}, t_{1}\right)$. Je suppose de plus, comme l'indique la figure, que lorsqu'on parcourt le contour de $E$ en laissant cet espace à droite on rencontre les côtés successifs dans l'ordre $T_{0}, U_{1}, T_{1}, U_{0}$.

Entourons les lignes droites $u_{0} u_{1}, t_{0} t_{1}$ de contours $C_{1}, C_{2}$ suffisamment rapprochés pour que les conditions suivantes soient satisfaites. Quand on 
fait varier $u$ et $t$ à l'intérieur de ces contours, la valeur de $x$, déduite de l'équation (3), qui est égale à $S_{0}$ pour $u=u_{0}, t=t_{0}$, reste fonction holomorphe de $u$ et de $t$. Tout pareillement, quand on fait varier $x$ et $u$ à l'intérieur des contours $C$ et $C_{1}$, la valeur de $t$ qui est égale à $t_{\mathrm{n}}$ pour $x=S_{0}, u=u_{0}$ reste une fonction holomorphe de $x$ et de $u$ que j'appellerai $\chi(x, u)$. Enfin, lorsque $x$ et $t$ restent compris à l'intérieur des contours $C$ et $C_{2}$, la valeur de $u$ qui est égale à $u_{0}$ pour $x=S_{0}, t=t_{0}$ reste holomorphe; soit $v=\phi(x, t)$ cette fonction. Les lignes

$$
u_{0} u_{1}, t_{0} t_{1}, T_{0}, U_{1}, T_{1}, U_{0}
$$

sont décrites dans le sens indiqué par les Hèches (fig. 4); sur chacune de ces lignes, il y a donc lieu de distinguer un bord droit et un bord gauche.

7. Posons, pour abréger,

$$
I I(x, u)=\int_{t_{0}}^{t_{1}} \frac{F(x, u, t) d t}{G^{n}(x, u, t)}
$$

$\Pi(x, u)$ est une fonction des deux variables indépendantes $x$ et $u$ qui admet, quand on regarde $x$ comme constant, un certain nombre de coupures variables avec $x$; ce sont les lignes obtenues en faisant varier $t$ de $t_{0}$ à $t_{1}$ dans l'équation (3). Je considère seulement celle de ces coupures qui rencontre la ligne $u_{0} u_{1}$ lorsque le point $x$ est situé dans l'espace $E$. En appelant $v_{0}$ et $v_{1}$ les extrémités de cette coupure mobile $\Gamma$, on aura, d'après la notation adoptée.

$$
v_{0}=\psi\left(x, t_{0}\right), \quad v_{1}=\psi\left(x, t_{1}\right) .
$$

Lorsque $x$ est à l'intérieur de $C$, mais à l'extérieur de $E, \Gamma$ ne rencontre pas $u_{0} u_{1}$, et rencontre cette droite en un seul point lorsque $x$ est à l'intérieur de $E$. De plus, d'après les hypothèses faites plus haut, cette ligne $\Gamma$ ne peut avoir de point double, tant que $x$ reste à l'intérieur de $C$; nous admettrons en outre qu'elle ne rencontre pas d'autre coupure.

La fonction $I(x, u)$ jouit évidemment des propriétés de la fonction étudiée au $\mathrm{n}^{\circ}$ 2. La différence des valeurs de $\Pi(x, u)$ en deux points infiniment voisins de $\Gamma$ est égale à $\pm 2 i \pi R(x, u), R(x, u)$ étant le résidu de

$$
\frac{F(x, u, t)}{G^{n}(x, u, t)}
$$


relatif au pôle défini par l'équation $G(x, u, t)=0$ quand on y considère $t$ comme la variable indépendante. Quand on considère $x$ comme constant, $\Pi(x, u)$ peut, dans le voisinage du point $v_{1}$ ettre mis sous la forme

$$
I I(x, u)=R(x, u) L\left(u-v_{1}\right)+P(u)
$$

$P(u)$ étant une fonction uniforme de $u$ qui admet le point $u=v_{1}$ pour póle d'ordre $n-$ I. Appelons $\lambda_{1}(x)$ le résidu relatif à ce pôle et $\lambda_{0}(x)$ le résidu relatif au pôle $v_{0}$. Il est clair, d'après la définition même de ces quantités, que $I(x, u), \lambda_{0}(x), \lambda_{1}(x)$ sont des fonctions uniformes à l'intérieur des contours $C$ et $C_{1}$.

8. Voyons ce qui arrive lorsque $x$ est sur le contour du quadrilatère $E$. Supposons $x$ sur le còté $T_{0}$; à une valeur de $x$ située sur ce côté correspond le couple de valeurs

$$
t=t_{0}, \quad u=u_{0}+\theta\left(u_{1}-u_{0}\right),
$$

$\theta$ ayant une valeur réelle comprise entre zéro et l'unité; on voit donc que, pour une pareille valeur de $x$, l'extrémité $v_{0}$ de $I$ vient coincider avec un point de la droite $u_{0} u_{1}$. Lorsque $x$ franchit $T_{0}$ en passant du bord gauche au bord droit ou de l'extérieur à l'intérieur de $E$, on sait, d'après une propriété bien connue des fonctions analytiques dont je me suis servi plusieurs fois dans le cours de ce travail, que le point $v_{0}$ passe pareillement $\mathrm{du}$ bord gauche au bord droit de $u_{0} u_{1}$. Si le point $x$ est situé sur le côté $U_{0}$, ̀̀ cette valeur de $x$ correspond le couple de valeurs

$$
u=u_{0}, \quad t=t_{0}+\lambda\left(t_{1}-t_{0}\right)
$$

il en résulte d'après la définition de la coupure que le point $u_{0}$ est sur $\Gamma$. On étudierait de même les autres cas, et la discussion est résumée dans le tableau suivant:

$$
\begin{aligned}
& x \text { traversant } T_{0}, \ldots \text { le point } v_{0} \text { traverse } u_{0} u_{1}, \\
& \ldots \ldots \ldots T_{1}, \ldots \ldots \ldots v_{1} \ldots \ldots \ldots \text { point } u_{0} \text { traverse } I \\
& \ldots \ldots \ldots U_{0}, \ldots \ldots \text { le } \\
& \ldots \ldots \ldots U_{1}, \ldots \ldots \ldots u_{1} \ldots \ldots
\end{aligned}
$$

Nous pouvons maintenant nous faire une idée très-nette de la façon dont 
se déplace la coupure $\Gamma$ avec $x$; les figures suivantes montrent tous les cas qui peuvent se présenter. J'ai figuré d'une part le chemin décrit par la variable, d'autre part les chemins décrits par les extrémités $v_{0}, v_{1}$.
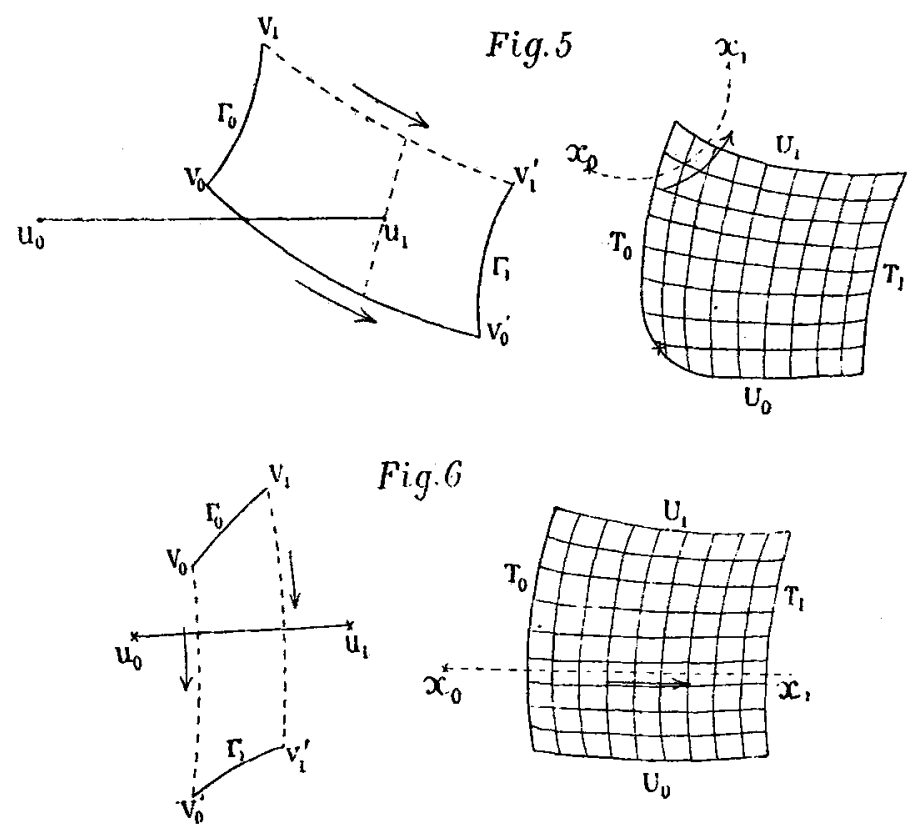

Fig. 6
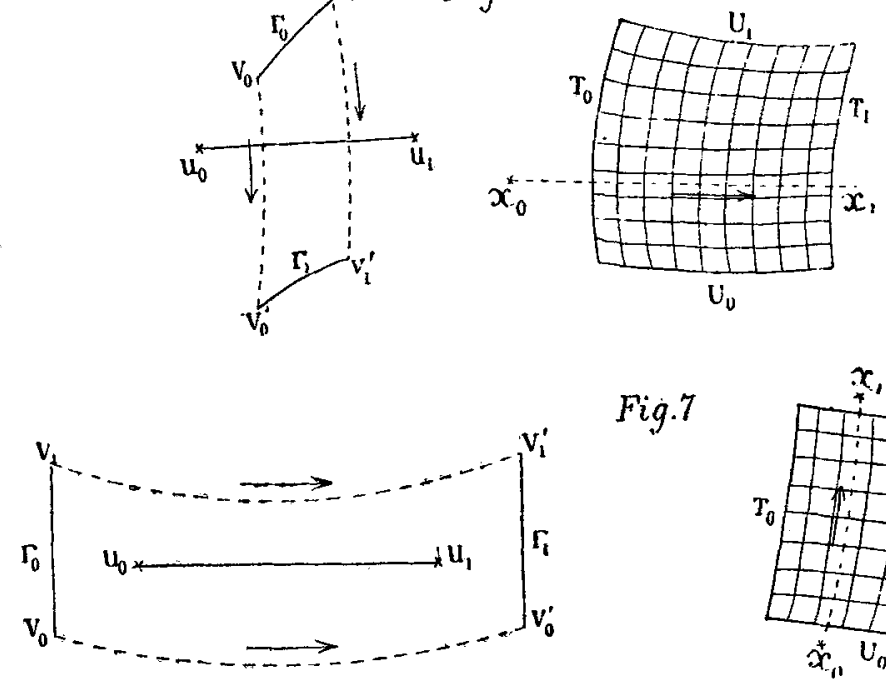

Fig.7

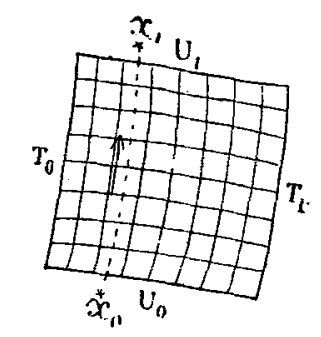

9. Imaginons que le chemin décrit par la variable pénètre à l'intérieur de $E$, et voyons ce que devient la fonction analytique qui est égale à $\Phi(x)$ à l'extérieur de ce quadrilatère. On reconnaít d'abord, en déformant infiniment peu les chemins d'intégration, ce qui entraine une déformation analogue des côtés $T_{0}, T_{1}, U_{0}, U_{1}$ que la fonction ne cesse pas d'exister. Mais, pour étudier les modifications qu'elle subit, il est nécessaire de distinguer plusieurs cas. Je suppose d'abord que la variable décrive un chemin pénétrant dans le quadrilatère $E$ par un certain côté, 
par exemple $T_{0}$, et ressortant par le même côté. La figure suivante montre le déplacement correspondant de $I$ :

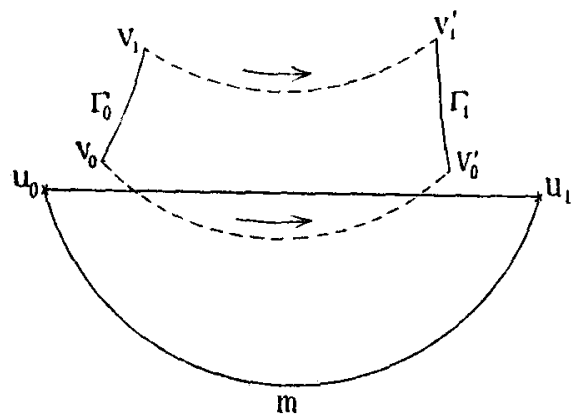

Fig. 8

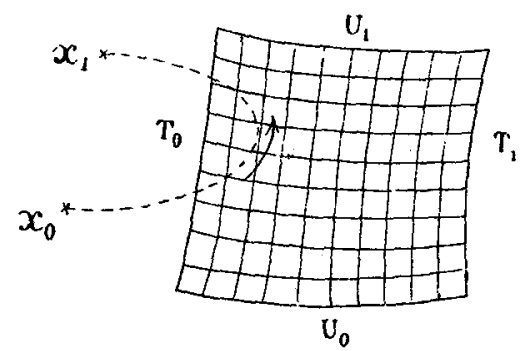

Avant que la coupure mobile atteigne la droite $u_{0} u_{1}$, on peut remplacer l'intégrale

$$
\int_{u_{0}}^{u_{1}} \Pi(x, u) d u
$$

par la même intégrale prise suivant le chemin $u_{0} m u_{1}$, qui n'est pas atteint par cette coupure. Lorsque le point $x$ sera venu en $x_{1}$ et la coupure $\Gamma$ en $\Gamma_{1}$, l'intégrale curviligne $\left(u_{0} m u_{1}\right)$ n'aura pas cessé d'avoir un sens et l'application du théorème de $\mathrm{CAUCHY}_{\mathrm{A}}$ montre que cette intégrale est encore égale à l'intégrale rectiligne $\left(u_{0} u_{1}\right)$; de sorte que la fonction est encore égale à $\Phi(x)$ aux environs du point $x_{1}$. Le même mode de raisonnement s'applique au côté $T_{1}$, et par raison de symétrie il en est évidemment de méme des côtés $U_{0}, U_{1}$; ce qu'on pourrait d'ailleurs démontrer directement en renversant l'ordre des intégrations. II résulte de l'étude qui vient d'être faite que les seuls chemins fermés qui puissent ne pas ramener $\Phi(x)$ à sa valeur initiale sont coux qui traversent deux côtés différents $\mathrm{du}$ quadrilatère; ce qui revient à dire que les seuls points singuliers de cette fonction à l'intérieur de $C$ sont les quatre sommets $S_{0}, S_{1}, S_{2}, S_{3}$.

IO. Il suffit évidemment de savoir ce qui arrive lorsque la variable traverse deux côtés consécutifs; je prends par exemple les côtés $T_{0}, U_{1}$; la figure suivante montre le déplacement correspondant de $\Gamma$.

Avant que la coupure $\Gamma$ n'ait atteint la ligne $u_{0} u_{1}$, remplaçons l'intégrale rectiligne $\left(u_{0} u_{1}\right)$ par l'intégrale curviligne $\left(u_{0} m n u_{1}\right)$, qui est 
égale à la précédente et qui ne cesse pas d'avoir un sens. Considérons la position $\Gamma_{2}$ occupée par $\Gamma$ un peu avant que $x$ n'ait atteint le cóté
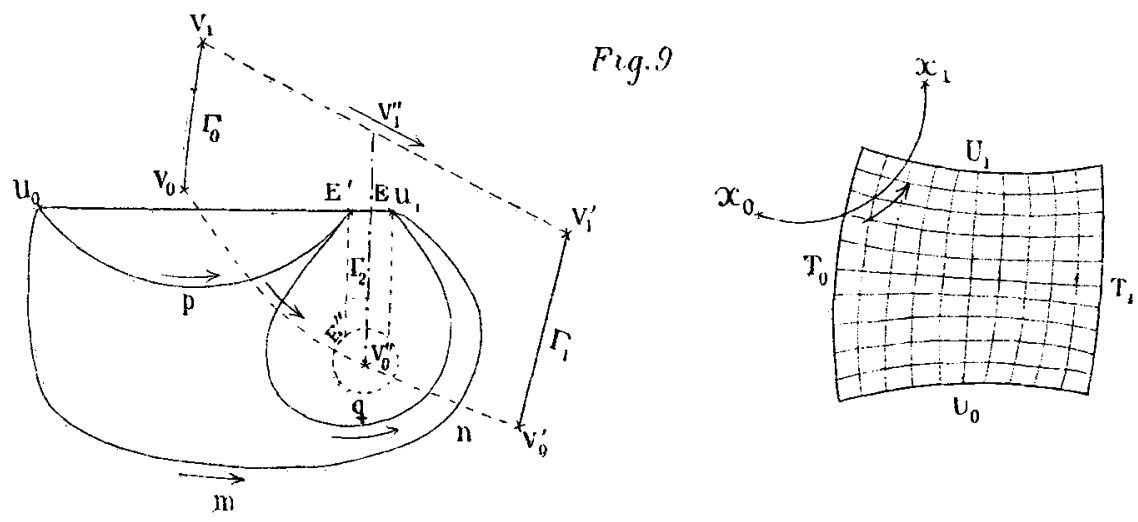

$U_{1} ; \Gamma_{2}$ coupe $u_{0} u_{1}$ en un point $e$ infiniment rapproché de $u_{1}$. Soit $e^{\prime}$ un nouveau point de cette ligne infiniment voisin de $e$. On peut encore remplacer $\left(u_{0} m n u_{1}\right)$ par la somme des deux intégrales $\left(u_{0} p e^{\prime}\right)+\left(e^{\prime} q u_{1}\right)$, dont la première peut être réduite à l'intégrale rectiligne $\left(u_{0} e^{\prime}\right)$. Le chemin $e^{\prime} q u_{1}$ peut à son tour être réduit à deux lignes infiniment rapprochées de la coupure $I_{2}$ et à un cercle de rayon infiniment petit $\rho$ décrit autour du point $v_{0}^{\prime \prime}$. Or, le long de la coupure $\Gamma, \Pi(x, u)$ prend de part et d'autre de cette ligne des valeurs dont la différence est $2 i \pi R(x, u)$; on aura donc

$$
\int_{\left(e^{\prime} q u_{1}\right)} I I(x, u) d u=2 i \pi \int_{e^{\prime}}^{e^{\prime \prime}} R(x, u) d u+\int_{(\rho)} I I(x, u) d u .
$$

Dans le voisinage du point $v_{0}$ on sait que $\Pi(x, u)$ est de la forme

$$
R(x, u) L\left(u-v_{0}\right)+P(u)
$$

l'intégrale

$$
\int_{(\rho)} P(u) d u
$$

est égale, d'après les notations adoptées, à $2 i \pi \lambda_{n}(x)$. Quant à l'intégrale

$$
\int_{(\rho)} R(x, u) L\left(u-v_{0}\right) d u,
$$


$R(x, u)$ étant holomorphe pour $u=v_{0}$, on vérifie facilement qu'elle est infiniment petite avec $\rho$. Soit en effet $f(u)$ une fonction holomorphe de $u$ dans le domaine d'un point $a$

$$
f(u)=\sum_{\nu=0}^{+\infty} A_{\nu}(u-a)^{\nu}
$$

posons

$$
F(u)=\sum_{\nu=0}^{+\infty} A_{\nu} \frac{(u-a)^{\nu+1}}{\nu+1}, \quad F_{1}(u)=\sum_{\nu=0}^{+\infty} A_{\nu} \frac{(u-a)^{\nu+1}}{(\nu+1)^{2}}
$$

Dans le domaine du point $a$, on aura

$$
\int f(u) L(u-a) d u=F(u) L(u-a)-F_{1}(u)+C,
$$

et cette intégrale, prise le long d'un cercle de rayon $\rho$, à partir d'un point $\alpha$ sera égale $\dot{a}$

$$
[F(u) L(u-a)]_{a}^{a},
$$

où à $2 i \pi F(\alpha)$; ce résultat est, comme on voit, infiniment petit avec $\rho$. On a donc, en supposant $\rho$ infiniment petit, c'est-à-dire $e^{\prime \prime}=v_{0}^{\prime \prime}$,

$$
\int_{\left(e^{\prime} q u_{1}\right)} \Pi(x, u) d u=2 i \pi\left[\int_{e^{\prime}}^{v_{0}^{\prime \prime \prime}} R(x, u) d u+\lambda_{0}(x)\right] .
$$

La coupure continuant à se déplacer de $\Gamma_{2}$ en $\Gamma_{1}$, la quantité contenue dans le second membre de l'égalité précédente conserve un sens, et quand $x$ sera venu en $x_{1}$ la continuation analytique de $\Phi(x)$ sera représentée par

$$
\left(u_{0} e^{\prime}\right)+2 i \pi\left[\int_{e^{\prime}}^{v_{0}} R(x, u) d u+\lambda_{0}(x)\right]
$$

où l'on doit maintenant faire $u_{1}-e^{\prime}$ infiniment petit; ce qui conduit à remplacer $\Phi(x)$ par

$$
\Phi(x)+2 i \pi \int_{u_{1}}^{v_{0}} R(x, u) d u+2 i \pi \lambda_{0}(x) .
$$

Les autres cas s'étudient de la même manière et conduisent à des résultats 
analogues. En résumé, la variable franchissant l'espace $E$, la fonction égale à $\Phi(x)$ au point de départ est égale au point d'arrivée ’̀ $\Phi(x)+2 i \pi \varphi(x)$, $\varphi(x)$ ayant l'une des valeurs suivantes, d'après le chemin décrit par la variable:

$x$ franchissant les côtés $T_{0}, U_{1}$, on a $\varphi_{1}(x)=\int_{u_{1}}^{v_{0}} R(x, u) d u+\lambda_{0}(x)$,

$$
\begin{aligned}
& \ldots \ldots \ldots \ldots \ldots U_{1}, T_{1}, \ldots \ldots \varphi_{2}(x)=\int_{v_{1}}^{u_{1}} R(x, u) d u+\lambda_{1}(x), \\
& \ldots \ldots \ldots T_{1}, U_{0}, \ldots \ldots \varphi_{3}(x)=\int_{u_{0}}^{v_{1}} R(x, u) d u-\lambda_{1}(x), \\
& \ldots \ldots \ldots U_{0}, T_{0}, \ldots \ldots \varphi_{0}(x)=\int_{v_{0}}^{u_{0}} R(x, u) d u-\lambda_{0}(\dot{x}) .
\end{aligned}
$$

Comme conséquences, on déduit de là que si on fait franchir à la variable $x$ les deux côtés $U_{1}, U_{0}$ successivement, $\Phi(x)$ se trouvera augmentée de $2 i \pi\left[\varphi_{2}(x)+\varphi_{3}(x)\right]=2 i \pi \int_{u_{0}}^{u_{1}} R(x, u) d u$. Si la variable franchit les còtés $T_{0}, T_{1}$ successivement, $\Phi(x)$ sera augmentée de

$$
2 i \pi\left[\varphi_{1}(x)+\varphi_{2}(x)\right]=2 i \pi\left[\int_{\tau_{1}}^{t_{0}} R(x, u) d u+\lambda_{0}(x)+\lambda_{1}(x)\right] .
$$

Ce dernier résultat est aisé à vérifier directement; on a vu plus haut que la coupure mobile ra de $\Gamma_{0}$ en $\Gamma_{1}$ (fig. Io), et l'intégrale rectiligne $\left(u_{0} u_{1}\right)$ est remplacée par l'intégrale $\left(u_{0} m u_{1}\right)$, qui est égale à l'intégrale rectiligne ou $\Phi(x)$, augmentée de l'intégrale

$$
\int \Pi(x, u) d u
$$

prise le long d'un contour $C$ environnant la coupure $\Gamma_{1}$ dans le sens direct. Ce dernier contour peut être réduit à deux circonférences de rayons infiniment petits ayant

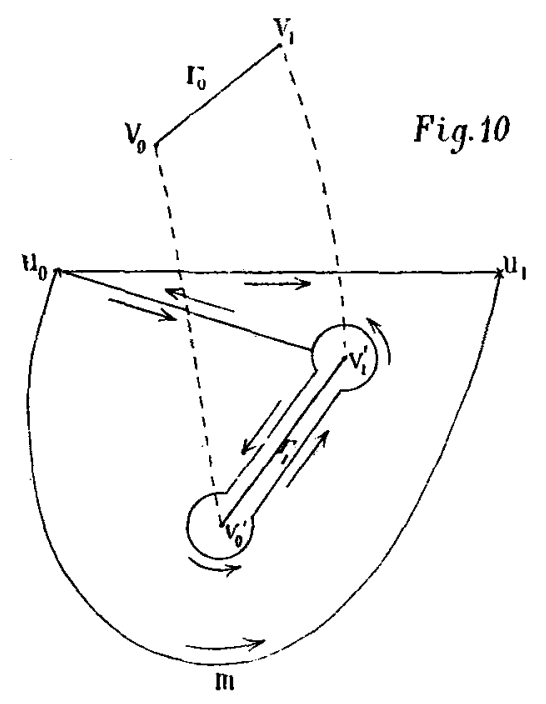


pour centres les points $v_{0}^{\prime}, v_{1}^{\prime}$ et à deux lignes infiniment rapprochées de la coupure. On trouve comme tout-à-l'heure que la partie de l'intégrale relative aux petites circonférences est égale $\dot{\mathrm{a}} 2 i \pi\left[\lambda_{0}(x)+\lambda_{1}(x)\right]$, et que ia partie provenant des autres lignes est $2 i \pi \int_{v_{1}}^{v_{0}} R(x, u) d u$.

II. Soit $n=\mathrm{I}$; on a alors

$$
R(x, u)=\frac{F(x, u, t)}{\frac{\partial G}{\partial t}},
$$

on supposant qu'on ait remplacé $t$ par sad valeur tirée de l'équation $G(x, u, t)=0$. Les résidus $\lambda_{0}(x), \lambda_{1}(x)$ sont nuls et les valeurs de $\varphi(x)$ se simplifient. Si la variable franchit les cótés $U_{1}, U_{0}, \Phi(x)$ se trouve augmentée de

$$
2 i \pi \int_{u_{0}}^{u_{1}} \frac{F^{\prime}(x, u, t)}{\frac{\partial G^{\prime}}{\partial t}} d u
$$

et si $x$ franchit les cótés $T_{0}, T_{1}, \phi(x)$ se trouve augmentée de

$$
2 i \pi \int_{n_{1}}^{v_{0}} \frac{F(x, u, t)}{\frac{\partial G}{\partial t}} d u .
$$

Ici une vérification s'offre d'elle-même. Supposons en eff'et qu'on ait effectué les intégrations dans l'ordre inverse; on aurait trouvé pour accroissement de $\Phi(x)$ lorsque $x$ franchit les côtés $T_{0}, T_{1}$,

On doit donc avoir

$$
2 i \pi \int_{i_{0}}^{t_{1}} \frac{F^{\prime}(x, u, t) d t}{\frac{\partial G}{\partial u}} .
$$

$$
\int_{t_{0}}^{t_{1}} \frac{F(x, u, t) d t}{\frac{\partial G}{\partial u}}=\int_{v_{v}}^{v_{1}} \frac{F(x, u, t) d u}{\frac{\partial G}{\partial t}} ;
$$


c'est en effet ce qui a lieu; car si dans la première intégrale on fait le changement de variable

$$
t=\chi(x, u),
$$

on retrouve identiquement la seconde. Ceci résulte de ce qu'aux limites $t_{0}, t_{1}$ pour $t$ correspondent pour $u$ les limites $v_{0}, v_{1}$ et de l'identité

$$
\frac{\partial G}{\partial u} d u+\frac{\partial G}{\partial t} d t=0 .
$$

I 2. Comme application, je considère l'intégrale double

$$
\Phi(x)=\int_{0}^{1} d u \int_{n}^{1} \frac{d t}{t+i u-x},
$$

qui rentre bien dans le cas de $n=\mathrm{I}$. Le quadrilatère $E$ se compose ici d'un carré ayant pour sommets les points d'affixes $\mathrm{O}, \mathrm{I}, i, \mathrm{I}+i$, (fig. I $\mathrm{I}$ ). Les diverses quantités qui figurent dans les formules ont respectivement les valeurs:

$$
\begin{gathered}
R(x, u)=\mathrm{I}, \quad \lambda_{0}(x)=\lambda_{1}(x)=\mathrm{o}, \\
v_{0}=-i x, \quad v_{1}=-i x+i ;
\end{gathered}
$$

par suite les $\varphi$ auront les valeurs suivantes:

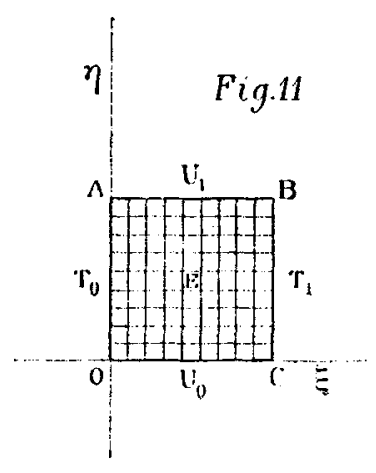

$$
\begin{array}{ll}
\varphi_{0}(x)=\int_{-i x}^{0} d u=i x, & \varphi_{1}(x)=\int_{1}^{-i x} d u=-i(x-i), \\
\varphi_{2}(x)=\int_{-i x+i}^{1} d u=i(x-\mathrm{I}-i), & \varphi_{3}(x)=\int_{0}^{-i x+i} d u=-i(x-1) .
\end{array}
$$

La vérification est immédiate, car un calcul élémentaire donne

$$
\Phi(x)=i[(x-\mathrm{I}-i) L(x-\mathrm{I}-i)+x L(x)-(x-\mathrm{I}) L(x-\mathrm{I})-(x-i) L(x-i)] .
$$

I 3. Dans le cas de $n=2$, les résidus $\lambda_{0}(x), \lambda_{1}(x)$ ne seront plus nuls en général et on trouve pour leurs expressions (voir $n^{\circ} 2$ )

$$
\lambda_{0}(x)=\frac{F^{\prime}(x, u, t)}{\frac{\partial G}{\partial u} \frac{\partial G}{\partial t}},
$$


où on a remplacé $t$ par $t_{0}$ et $u$ par $v_{0}$, et pour $\lambda_{1}(x)$ une expression analogue. Soit, par exemple,

$$
\Phi(x)=\int_{0}^{1} d u \int_{0}^{1} \frac{d t}{(t+i u-x)^{2}} ;
$$

le quadrilatère $E$ est le mème que tout-à-l'heure. On a ici

$R(x, u)=0, \quad \lambda_{0}(x)=-i, \quad \lambda_{1}(x)=i, \quad v_{0}=-i x, \quad v_{1}=-i x+i ;$

et par suite:

$$
\varphi_{0}(x)=\varphi_{2}(x)=i, \quad \varphi_{1}(x)=\varphi_{3}(x)=-i .
$$

Les résultats sont encore faciles à vérifier, car on trouve

$$
\Phi(x)=i L\left[\frac{x(x-1-i)}{(x-i)(x-1)}\right] .
$$

Remarque. Chacune des fonctions $\varphi_{0}(x), \varphi_{1}(x), \varphi_{2}(x), \varphi_{3}(x)$ étant holomorphe à l'intérieur de $C$, la différence

$$
\Phi(x)-\sum_{i=0}^{i=3} \varphi_{i}(x) L\left(x-S_{i}\right)
$$

sera, d'après ce qui précède, une fonction uniforme $\Psi(x)$ à l'intérieur du même contour, qui ne peut présenter de discontinuité quaux points $S_{0}, S_{1}, S_{2}, S_{3}$. Par une méthode analogue à celle qui a été employée au $n^{\circ} 3$, on reconnait que chacun de ces points est un pôle d'ordre $n-2$ pour $\Psi(x)$; dans les deux cas particuliers de $n=1$ et de $n=2$, ces points sont des points ordinaires: ce qui est bien d'accord avec les résultats précédents.

14. Soit $f(t)$ une fonction rationnelle de $t$ dont les coefficients ne contiennent ni $u$ ni $x$ et qui n'admet, pour fixer les idées, que des póles simples:

$$
f(t)=\frac{R_{1}}{t-p_{1}}+\frac{R_{s}}{t-p_{s}}+\ldots+\frac{R_{n}}{t-p_{n}} .
$$

Considérons l'intégrale double

$$
\Phi(x)=\int_{-\infty}^{+\infty} d u \int_{0}^{2} f(u+t-x) d t
$$


où les intégrations sont effectuées pour $u$ le long de l'axe réel, et pour $t$ le long d'une ligne droite joignant l'origine au point d'affixe $l=\alpha+\beta i$. Soit $p=a+i b$ un póle de $f(t)$; l'intégrale double précédente n'aura aucun sens pour toutes les valeurs de $x$ à l'intérieur de la bande indéfinie comprise entre deux parallèles à l'axe réel menées par les points d'affixes $p$ et $p+l$. Soient

$$
p_{1}=a_{1}+i b_{1}, \quad p_{2}=a_{2}+i b_{2}, \ldots, p_{n}=a_{n}+i b_{n},
$$

les póles de $f(t)$, rangés par ordre des coefficients de $i$ croissants, et supposons que la différence $b_{i+1}-b_{i}$ soit toujours supérieưre à $\beta$. A chacun de ces pôles correspondra une bande et deux bandes consécutives n'empiéteront jamais l'une sur l'autre. Le plan se trouve ainsi divisé en $n+$ I parties non connexes entre elles. Ou reconnaît d'abord que $\Phi(x)$ est constant dans chacune de ces parties; si on l'écrit en effet

$$
\Phi(x)=\int_{0}^{l} d t \int_{-\infty}^{+\infty} f(u+t-x) d u
$$

on aura

$$
\Phi^{\prime}(x)=-\int_{0}^{l} d t \int_{-\infty}^{+\infty} f^{\prime}(u+t-x) d u
$$

$f(\infty)$ étant nul, comme il est nécessaire de le supposer, on voit que l'intégrale

$$
\int_{-\infty}^{+\infty} f^{\prime}(u+t-x) d u
$$

est nulle pour toute valeur de $x$ et de $t$ et par suite on a $\Phi^{\prime}(x)=0$. Mais cette constante sera variable d'un intervalle à une autre. En premier lieu, pour savoir sa valeur lorsque $x$ est dans la partie au-dessous de la première bande ou au-dessus de la dernière, on peut faire $x=\infty$; ce qui donne $\Phi(x)=0$. Pour calculer l'accroissement de $\Phi(x)$ lorsque la variable traverse la bande correspondant au póle $p_{i}$ en passant de l'intervalle situé au-dessous à l'intervalle situé au-dessus, on peut, soit considérer ce cas comme limite du cas général, soit faire le raisonnement 
directement, et on trouve pour valeur de cet accroissement $2 i \pi \int_{i_{0}}^{n_{1}} R_{i} d u$. Ici $v_{0}=x, v_{1}=x-l$, et par suite

$$
\int_{v_{0}}^{v_{1}} R_{i} d u=-l R_{i}
$$

Il en résulte que dans le premier intervalle on aura $\Phi(x)=-2 i \pi l R_{1}$, dans le second $\Phi(x)=-2 i \pi l\left(R_{1}+R_{2}\right), \ldots$ ct enfin au dessus de la dernière coupure $\Phi(x)=-2 i \pi l\left(R_{1}+R_{2}+\ldots+R_{n}\right)$. Comme on doit avoir dans cette partie du plan $\Phi(x)=0$, on retrouve la condition connue

$$
R_{1}+R_{2}+\ldots+R_{n}=\text { ○. }
$$

En remplaçant les constantes $R$ par des fonctions de $x$ on parvient à l'expression, sous forme d'intégrale double, d'une fonction qui coïncide avec $n$ - I fonctions données tout-à-fait arbitraires à l'intérieur de $n$ - I intervalles séparés par des bandes de largeur finie, et non par de simples coupures. (Voir Hermite, Sur quelques points de la théorie des fonctions. Journal de BorchardT, T. 9 I, p. 69.)

Des faits analogues ont lieu apparemment pour des intégrales définies multiples à un nombre quelconque de variables. Le quadrilatère $E$ sera remplacé, autant que j'ai pu le voir, par un polygone d'un nombre $2^{n}$ de cótés pour les intégrales multiples d'ordre $n$.

Les principaux résultats contenus dans ce travail ont paru dans une note, présentée à l'Académie des Sciences le 30 Avril 1883.

Toulouse, Octobre 1883 . 